\title{
Analysis of Factors Regulating Erythrocyte Deformability
}

\author{
Narla Mohandas, Margaret R. Clark, Mark S. Jacobs, and Stephen B. Shohet, \\ Cancer Research Institute and the Departments of Laboratory Medicine and \\ Medicine, University of California, San Francisco, California 94143
}

A в S T R A C T Using a laser diffraction technique, we have studied factors that influence the deformability of erythrocytes. Variations in suspending medium osmolality and applied shear stress were employed to isolate the individual contributions to whole cell deformability of internal viscosity, surface area-to-volume ratio, and viscoelastic properties of the membrane. An experimental system was devised in which normal cells were modified in vitro to induce specific alterations in each factor. Measurements of deformability as a function of medium osmolality showed characteristic behavior of the modified cells. Reduced surface area-to-volume ratio was detected by an exaggeration of the normal decrease in deformability as medium osmolality was decreased. In contrast, increased internal viscosity was detected by an increase in deformability as osmolality was decreased. Finally, decreased membrane flexibility was detected by reduced deformation at low shear stress.

These methods of analysis were applied to cells from patients with hereditary spherocytosis, hereditary pyropoikilocytosis, and hemoglobin CC disease to define the basis of reduced deformability. Hereditary spherocytes showed the combined effects of reduced surface area and increased internal viscosity. Hereditary pyropoikilocytes revealed the effects of severely reduced surface area-to-volume ratio. Hemoglobin CC cells showed only the effects of high internal viscosity. An increase in the membrane shear modulus (decreased membrane deformability) was not evident in these disorders.

\section{INTRODUCTION}

Erythrocyte deformability, or the capacity of the cell to change its shape under applied stress, is important for erythrocyte function. Because the small vessels of the microcirculation have a diameter less than that of the resting erythrocyte, cells must deform markedly

Part of this material has been presented previously in abstract form in 1979. Blood. 54:(Suppl. 1) 30a.

Received for publication 3 October 1979 and in revised form 29 April 1980. as they circulate. Reduced erythrocyte deformability plays an important role in shortened erythrocyte survival in many types of hemolytic anemia (1-4). Several methods are available to measure erythrocyte deformability. These include filtration through small pore filters, viscometry of cell suspensions, aspiration of cells into micropipets, and deformation of individual cells under fluid shear stress (5-12). However, each technique is based on a particular criterion for cell deformability that may involve only one aspect of cell deformability behavior. For example, the filtration method measures the ability of cells to enter or pass through openings smaller than the resting diameter of the cell. In contrast, bulk viscometric techniques test cells on the basis of a reduction in the bulk viscosity of the suspension with increasing shear stress. The micropipet technique evaluates erythrocyte deformability on the same basis as filtration, but is applied to one cell at a time and under defined stress conditions.

The common property of cells that underlies these and other methods for deformability measurement is the ability of erythrocytes to alter their shape in response to applied shear stress, whether it is imposed by a stationary barrier or by the momentum of viscous fluid flow. To represent the physiologically relevant capacity of cells to change shape under shear stress in the circulation, we defined whole cell deformability in our in vitro model as the extent of cell elongation in response to a steady-state application of fluid shear stress. When defined in this manner, whole cell deformability is primarily regulated by three separate cellular properties: the internal viscosity, the surface area-to-volume ratio $(\mathrm{S} / \mathrm{V}),{ }^{1}$ and the viscoelastic properties of the membrane $(2,4,13)$. The various methods used to measure deformability have different sensitivities to each of these three factors. For example, filtration techniques are very sensitive to reduced $\mathrm{S} / \mathrm{V}$ (depending on the filter pore size) and less sensitive

${ }^{1}$ Abbreviations used in this paper: cp, centipoise; DI, deformability index; $\mathrm{DI}_{\max }$, maximum deformation; LPC, lysolecithin; MCHC, mean cell hemoglobin concentration; PBS, phosphate-buffered saline; S/V, surface area-to-volume ratio. 
to increased internal viscosity. Bulk viscometric methods are strongly influenced by cell/cell interactions and are less sensitive to the determinants of reduced deformability for individual cells (13). The sensitivity to each of the three factors in the micropipet method depends on the size of the pipet opening. Measurements with small pipets $(<1.0 \mu \mathrm{m})$ are influenced primarily by the membrane shear modulus. Measurements with larger pipets are influenced by the effects of increased internal viscosity and reduced S/V as well (9). Observation of cells attached to surfaces and then subjected to fluid shear stress provides information primarily about membrane properties (13).

With each of the techniques described, reduced deformability has been demonstrated in a variety of hemolytic disorders. However, because of the various differences in those techniques, correlations between measured deformability and cell survival are qualitative, rather than quantitative. Furthermore, none of the methods for measuring deformability have previously been used to distinguish the mechanisms regulating the deformation of individual cells or to define their physiologic influence.

Over the last few years, we have used still another method to measure cell deformability, employing an instrument called the ektacytometer. In this instrument, cells in a dilute suspension in a viscous medium are subjected to a laminar fluid shear stress field. In response to the applied stress, the resting discoid shape of normal erythrocytes is deformed into a shape best described as a prolate ellipsoid (14). Laser diffraction patterns of cells in their resting and deformed states are then measured to obtain quantitative information concerning the dimensions of the corresponding cells. The changes in cell dimensions with increasing shear stress thus provide a measure of cell deformability. This instrument has been used in a semiquantitative way to demonstrate reduced deformability in several hemolytic disorders (15). Recently, the use of a new image analysis system has allowed us to obtain reproducible quantitative data from the laser diffraction patterns. The measurements using this new system have been found to correlate with direct measurements of diffraction pattern dimensions that, in turn, are related to the extent of cell deformation (16).

With the new analysis system we have found that the quantitative deformation of erythrocytes at varying applied shear stresses and varying suspending medium osmolalities can distinguish the influence of each of the three major cellular factors regulating whole cell deformability. In preliminary demonstration studies, we have applied these techniques to the analysis of cell deformability in three hemolytic disorders: hereditary spherocytosis, hemoglobin CC disease, and hereditary pyropoikilocytosis. In hereditary spherocy- tosis, reduced $\mathrm{S} / \mathrm{V}$ plays a dominant role in reducing cell deformability, with an additional influence of increased internal viscosity in a subpopulation of cells with high mean cell hemoglobin concentration (MCHC). In hemoglobin CC disease, increased internal viscosity alone causes reduced deformability, and its influence is expressed in all cell subpopulations. In hereditary pyropoikilocytosis, severely reduced $\mathrm{S} / \mathrm{V}$ is responsible for most of the deformability loss.

\section{METHODS}

The ektacytometer. The ektacytometer has been described in detail previously $(14,17)$. It comprises a concentric cylindrical viscometer in which the outer cylinder can be rotated to produce a well-defined varying shear stress field. A laser beam that is deflected through the cell suspension contained in a $0.5-\mathrm{mm}$ gap between the two cylinders is diffracted by the cells to produce the patterns from which deformation is determined. A schematic diagram of the instrument, with the new data analysis system, is shown in Fig. 1. In this system, a quantitative assessment of the cell deformability is derived from photometric measurement of the light intensity at selected points on the diffraction pattern, designated as $A$ and $B$ in the diagram. $A$ and $B$ are chosen to be near the periphery of the inner diffraction ring of a stationary erythrocyte suspension. They are positioned on the major and minor axes, respectively, of the elliptical pattern produced by deforming cells. Therefore, as cell deformation increases, the light intensity at point $A$ increases and at point B decreases. A mechanical chopper permits the use of a single photomultiplier to measure the

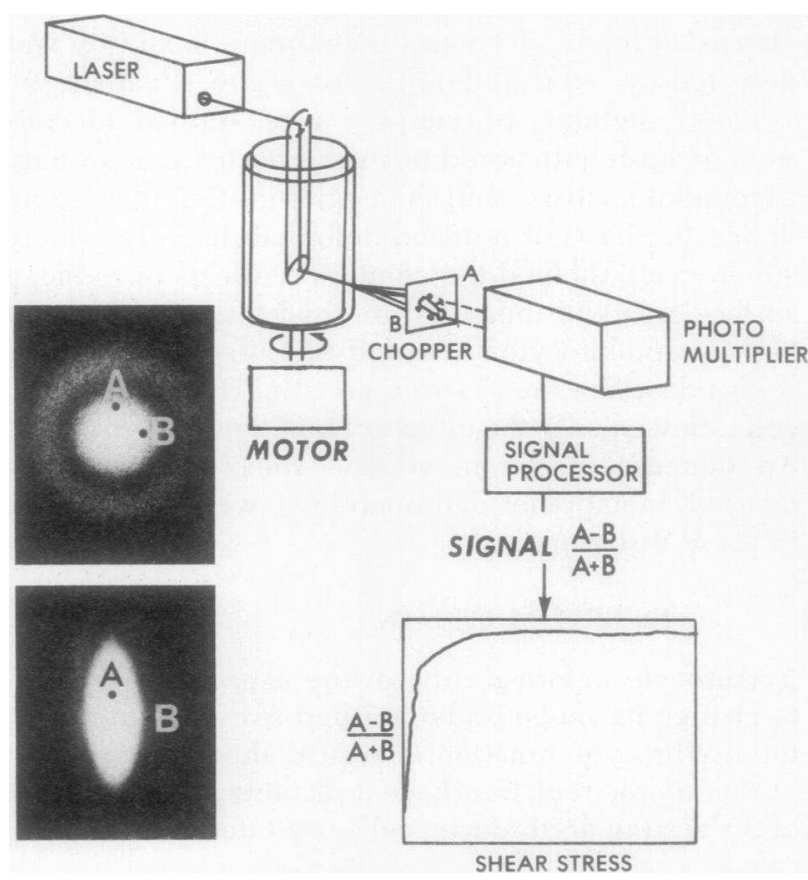

FIGURE 1 Schematic diagram of the ektacytometer with automatic image analysis system. Points $A$ and $B$, at which the signal is photometrically monitored, are indicated on the diffraction images. 
light intensity at points A and B. An electronic comparator circuit then generates a signal that is proportional to the quantity $A-B / A+B$, where $A$ and $B$ are the light intensity at points $A$ and $B$, respectively. This quantity is recorded as a function of linearly increasing shear rate. The signal is zero for nondeforming cells that maintain a circular diffraction pattern at all values of applied shear stress. On the other hand, for deforming cells that produce a progressively elongated pattern with increasing shear stress, the signal increases to a plateau value that represents the maximum deformation. As will be discussed later, the variation of $A-B / A+B$ with shear stress is the same as the variation in ellipticity of the diffraction pattern when measured optically. Because the dimensions of the diffraction pattern provide a measurement of the cell dimensions (16), the $A$ - B/A + B signal also provides the same information.

The medium used to dissolve the dextran was phosphatebuffered saline (PBS; $10 \mathrm{mM}$ sodium phosphate, $\mathrm{pH} 7.4$ ), in which the concentration of $\mathrm{NaCl}$ was adjusted to give the desired final osmolality. In the present experiments, dextran from Sigma Chemical Co. (St. Louis, Mo.) (40,000 average mol wt), and from Pharmacia Fine Chemicals (Div. of Pharmacia, Inc., Piscataway, N. J.) (dextran T40) was used. The primary difference between these two materials was in their osmotic strength. The Sigma dextran contributed 55 $( \pm 3) \mathrm{mosmol} / \mathrm{kg}$ at a concentration of $25 \mathrm{~g} / 100 \mathrm{ml} \mathrm{PBS}$; the more rigorously isolated Pharmacia product contributed 20 $( \pm 3) \mathrm{mosmol} / \mathrm{kg}$ at the same concentration. The increase in osmotic strength over that expected for pure $40,000 \mathrm{~mol}$ wt polymer $(6 \mathrm{mosmol} / \mathrm{kg})$ is the result of the presence of appreciable quantities of lower molecular weight polymer that inevitably accompany the ethanol-precipitated material (product specifications, Pharmacia and Sigma). The osmotic contribution of each product was taken into account in preparing dextran solutions of various osmotic strengths. Osmolality was measured using a vapor pressure osmometer (Wescor, Inc., Logan, Utah).

In most experiments, as described in Results, $1.5 \times 10^{8}$ cells were suspended in $3 \mathrm{ml}$ of dextran solution immediately before measurements were made $(\sim 30 \mu$ l of $40 \%$ hematocrit suspensions). However, even when cells were allowed to stand in isotonic dextran solutions for periods up to $4 \mathrm{~h}$, no changes in cell deformability were found.

Modification of deformability in normal erythrocytes. To modify erythrocyte deformability in specific ways with respect to the three major factors that regulate cell deformability, four experimental maneuvers were employed. First, to produce cells with reduced $S / V$, erythrocytes were treated with lysolecithin (LPC, Sigma Chemical Co.). In the concentration range used ( $1-5 \mu \mathrm{mol} \mathrm{LPC} / \mathrm{ml}$ cells), LPC results in the conversion of discocytic erythrocytes into echinocytes III up to prelytic spherocytes (18). The latter morphologic stages are characterized by a "budding off" and loss of membrane surface, with virtually no change in cell volume or MCHC and, hence, with little change in internal viscosity. After incubation in the presence of LPC for $5 \mathrm{~min}$ at room temperature, the cells were washed in isotonic PBS containing $1 \%$ bovine serum albumin (Sigma Chemical Co., Fraction V). This removed the LPC and caused the cells to revert to a stomatocytic morphology. In these experiments, changes in cell $\mathrm{S} / \mathrm{V}$ were confirmed by measurements of osmotic fragility.

Second, to produce cells with increased internal (cytoplasmic) viscosity, erythrocytes were dehydrated, using nystatin and sucrose as previously described (19). This procedure involved the addition of a stock solution of nystatin $(5 \mathrm{mg} / \mathrm{ml}$ methanol) to a $5 \%$ suspension of erythrocytes in a medium whose $\mathrm{Na}$ and $\mathrm{K}$ concentrations were those found in the interior of normal cells ( $\mathrm{Na}: 12 \mathrm{meq} / \mathrm{liter}$ cell water; $\mathrm{K}: 155$ meq/liter cell water). The final nystatin concentration was 30 $\mu \mathrm{g} / \mathrm{ml}$ of suspension. The presence of the antibiotic allowed the equilibration of $\mathrm{Na}$ and $\mathrm{K}$ across the cell membrane. Subsequent addition of sucrose to give a final osmolality of $350,400,450$, or $500 \mathrm{mosmol} / \mathrm{kg}$ then promoted overall efflux of cations and water from the permeable cells. After $20 \mathrm{~min}$ of incubation at $0^{\circ} \mathrm{C}$, the nystatin was washed out of the membrane by solutions identical to the suspending medium, but without nystatin. It was necessary to use the wash solutions at $37^{\circ} \mathrm{C}$ to remove the nystatin and to restore normal permeability characteristics to the cells. Finally, the cells were washed and resuspended in isotonic PBS before measurement of deformability. Cell dehydration was confirmed by measurement of osmotic fragility. In addition, homogeneity of the dehydrated cells was demonstrated by density gradient centrifugation (vide infra).

Finally, two methods were used to produce cells with reduced membrane deformability (increased shear modulus). In the first, PBS-washed erythrocyte suspensions were heated from $47^{\circ}$ to $48^{\circ} \mathrm{C}$ for periods of time ranging from 2 to $30 \mathrm{~min}$. This gentle heating, which did not alter cell shape or cause fragmentation, primarily increased the membrane shear modulus (20) and had only a minimal influence on the MCHC. In these experiments, measurement of osmotic fragility demonstrated the absence of substantial change in cell water.

The second method involved treatment of washed cells with diamide at concentrations of $0.1-0.5 \mathrm{mM}$. After resuspension in diamide-containing PBS, the cells were incubated at $37^{\circ} \mathrm{C}$ for $60 \mathrm{~min}$. They were then washed two times and resuspended in PBS. As shown by Fischer et al. (21), treatment with low concentrations of diamide causes an increase in membrane shear modulus without perturbing the normal shape or volume. To confirm this, we measured osmotic fragility after diamide treatment and found no appreciable changes.

Cell separation. To assess the deformability of various cell populations in the potentially heterogeneous cell mixture in blood from patients, the blood was separated on Stractan (St. Regis Paper Co., Tacoma, Wash.) density gradients as previously described (22). Linear continuous gradients with a density range of $1.070-1.124 \mathrm{~g} / \mathrm{ml}$ were formed above a $1.140-\mathrm{g} / \mathrm{ml}$ dense Stractan cushion. After centrifugation at $50,000 \mathrm{~g}$ for $30 \mathrm{~min}$, the various subpopulations were removed and washed three times in PBS before deformability measurements were made.

For some studies, cells were separated by spinning samples of packed, unwashed erythrocytes in a microhematocrit centrifuge (23). First, the cells were sedimented at $8,000 \mathrm{~g}$ for $5 \mathrm{~min}$, and were then drawn into $200-\mu \mathrm{l}$ capillary tubes. The tubes were then sealed with a flame, and then spun at $11,000 \mathrm{rpm}$ for $10 \mathrm{~min}$ in a microhematocrit centrifuge at room temperature. The tubes were scored and broken to obtain the desired subpopulations of cells along the length of the packed cell column.

\section{RESULTS}

Characterization of the deformation curve. As normal cells deformed under increasing shear stress, the image analysis system of the ektacytometer produced a continuous deformation curve. The signal $A-B / A+B$, which we define as the deformability index (DI), increased with increased applied shear stress $($ shear stress $=$ shear rate $\times$ suspending medium 
viscosity). To validate the DI signal as a measure of actual cell deformation, we compared it with two other more direct measurements. First, we measured the actual dimensions of the ektacytometric diffraction patterns photographed at various levels of shear rate. From the dimensions we then calculated the pattern ellipticity $L-W / L+W$, where $L$ and $W$ are the lengths of the major and minor axes of the diffraction pattern. Second, we compared both DI and the pattern ellipticity to the cellular ellipticity $\mathrm{E}^{\prime}$, measured by Fischer et al. (24) from photographic images of individual cells subjected to shear stress in the rheoscope in media of comparable viscosities. These three different measurements of deformation are plotted as a function of shear rate in Fig. 2. Note the close correspondence of the discrete values for both the pattern and cell ellipticity with the continuous DI curve over the entire shear rate range up to $1,000 \mathrm{~s}^{-1}$. This close correspondence indicates that the DI function accurately measures cell deformation.

Experiments conducted at suspending medium viscosities $>20$ centipoise $(\mathrm{cp})$ indicated that the DI function and the ellipticity function were the same only at shear stress values below $75 \mathrm{dyn} / \mathrm{cm}^{2}$. Above this value, the cells and their diffraction pattern

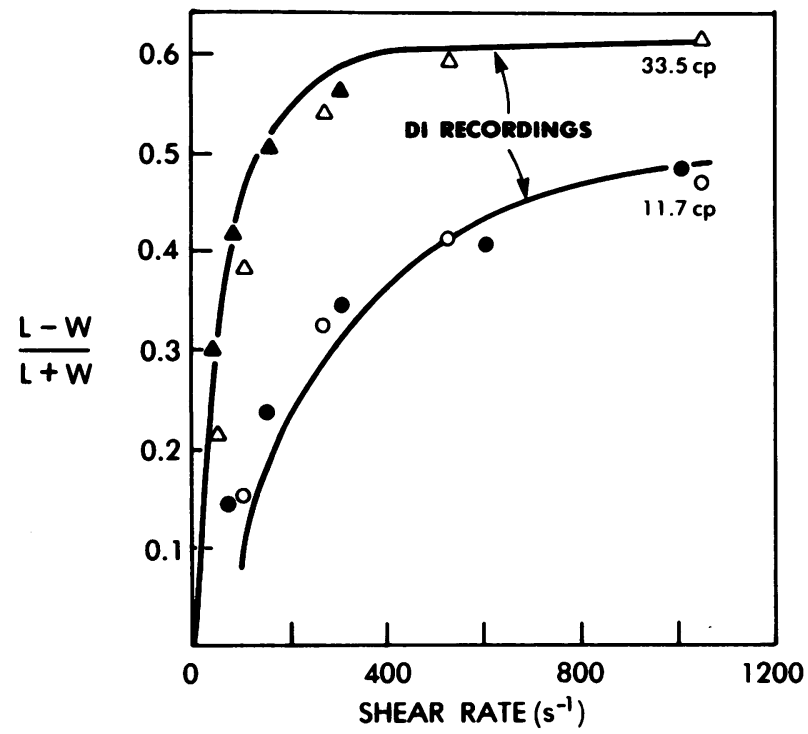

FIGURE 2 Various measurements of cell deformation as a function of applied shear rate at two different suspending medium viscosities. The open symbols represent the ellipticity $(\mathrm{L}-\mathrm{W} / \mathrm{L}+\mathrm{W})$ of ektacytometer diffraction patterns, calculated from the major (L) and minor (W) axes dimensions measured with photographic negatives. The closed symbols represent the ellipticity of actual deforming cells that Fischer et al. (24) measured by direct observation in the rheoscope. The continuous lines are the recorded DI curves from the image-analysis system of the ektacytometer. Note the close agreement between all three measurement methods. $O \Delta$ diffraction pattern ellipticity; $\boldsymbol{\Delta}$, cell ellipticity (Fischer et al.). showed additional slight elongation. The DI function, however, appeared to reach its maximum value at an applied shear stress value of $75 \mathrm{dyn} / \mathrm{cm}^{2}$ and showed no further increase beyond this point.

An additional test of the validity of the DI function as a measure of cell deformation was provided by plotting this function in a form suggested by Fischer et al. (24). Fig. 3 shows data obtained for cells from two normal donors, using a range of suspending media from 10.8 to $34.8 \mathrm{cp}$, plotted as DI vs. $\log \left(\eta^{1.5} \times\right.$ shear rate), where $\eta$ is the suspending medium viscosity. In this plot, the points derived from the various DI curves fall along a single S-shaped curve, within the outlines given by Fischer's data (solid lines) based on actual cell dimensions.

Deformability measurements in the ektacytometer require the use of a viscous suspending medium. We wanted to be certain that the dextran used to increase viscosity influenced cell deformation only through its viscosity contribution, and not through any alteration of cellular properties. As a test for this second condition, we performed ektacytometric measurements using other polymeric substances: a higher molecular weight dextran $(170,000$ average mol wt), Ficoll $(400,000$ average mol wt), and polyvinyl pyrolidine $(360,000$ average mol wt). For any given suspending medium viscosity, the deformation curves obtained in these media for cells from a normal individual could be superimposed at all shear stress values.

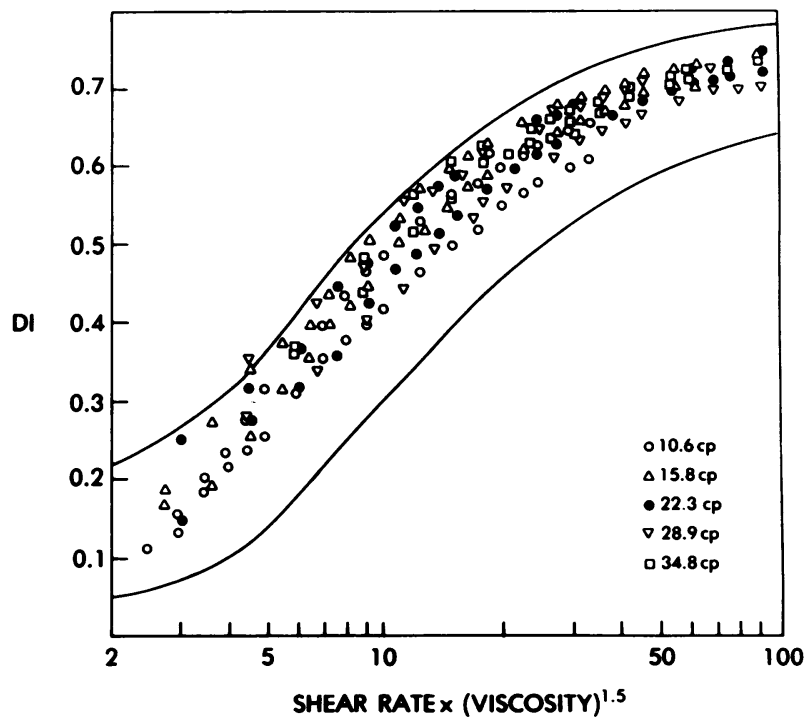

Figure 3 A plot of DI vs. the quantity shear rate $\times$ (viscosity) ${ }^{1.5}$ for five different suspending medium viscosities. Each point was taken from the continuous DI curves of ektacytometric measurements. The continuous curves represent the envelope of rheoscope data plotted in the same form by Fischer et al. (24). Note that all the data from the ektacytometer fall along the single S-shaped curve enclosed by the envelope. $O, 10 \mathrm{cp} ; \Delta, 15.8 \mathrm{cp} ; 0,22.3 \mathrm{cp} ; \nabla, 28.9 \mathrm{cp} ; \square, 34.8 \mathrm{cp}$. 
The comparison of DI and the diffraction pattern ellipticity established the quantitative relationship between DI and the extent of average elongation of a population of deforming cells. It was also important to know whether the DI signal provided a quantitative measure of the proportion of deformable cells in a mixed population of deformable and undeformable cells. To answer this question, we performed ektacytometric measurements on glutaraldehyde fixed and normal cells mixed in varying proportions. Fixed cells did not deform, but gave a constant zero value for DI over the entire shear stress range. Mixtures of fixed and unfixed cells produced DI curves intermediate between this base line and the normal DI curve. Furthermore, a plot of the maximum deformation $\left(D I_{\max }\right)$ vs. the percentage of unfixed cells was linear (Fig. 4).

To define the optimum conditions for reproducible measurements of deformability, we studied the deformation curve at several dextran concentrations and over a range of cell concentrations. We found that the shape of the DI curve changed upon increase of dextran concentration from $10 \mathrm{~g} / 100 \mathrm{ml}$ PBS up to 25 g/100 ml PBS (Fig. 5). In the least concentrated solution, whose viscosity was $5.2 \mathrm{cp}$, the cells appeared simply to orient with respect to the shear stress field. They never actually deformed to produce the characteristic elliptical diffraction pattern. At higher dextran concentrations of 15-25 g/100 ml PBS, the cells deformed over the entire shear stress range, with increasing deformation in the more viscous solutions for a given shear rate. However, even in $15 \mathrm{~g} / 100 \mathrm{ml}$ dextran $(10.2$ cp viscosity), the older, most dense $10 \%$ of normal cell populations did not deform appreciably. At 25 $\mathrm{g} / 100 \mathrm{ml}$, the medium viscosity $(21.6 \mathrm{cp})$ was high enough to cause virtually uniform deformation of all the cells from normal blood samples. This was confirmed by measurement of subpopulations from Stractan density gradients, in which only the most dense $1 \%$ of the cells exhibited reduced deformability.

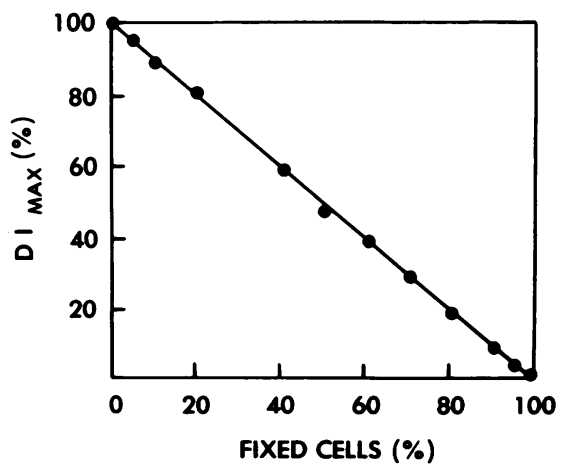

FIGURE $4 \mathrm{DI}_{\max }$ decreases linearly with increasing percentages of fixed cells.

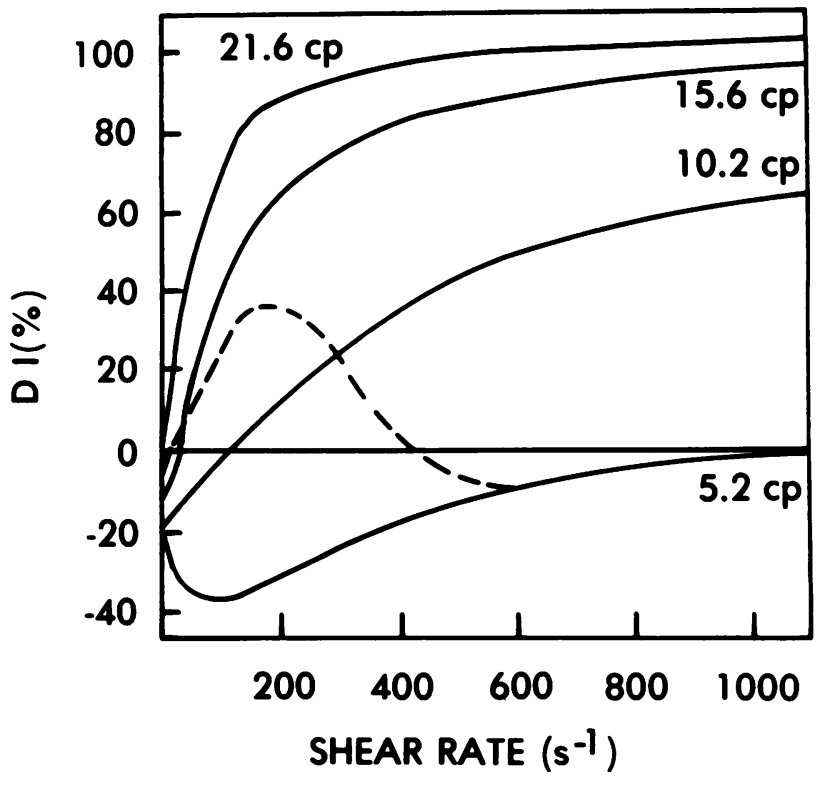

Figure 5 DI vs. shear rate for normal cells in dextran medium of varying viscosities. Note the use of shear rate rather than shear stress on the abscissa. (Shear stress = shear rate $X$ viscosity.) The dashed line was obtained as shear rate was increasing; all the solid lines were recorded with decreasing shear rate. For viscosities $>10 \mathrm{cp}$, curves for increasing and decreasing shear rates were nearly identical. At the lower viscosity, differing orientation of the cells caused divergence of the two curves.

Thus, subsequent experiments were performed in 25 $\mathrm{g} / 100 \mathrm{ml}$ PBS dextran solutions to minimize any effects of normal variation in MCHC and internal viscosity.

The effects of varying cell concentration are summarized in Fig. 6. The plateau amplitude of the DI showed a maximum value in the range of 0.4 to $1.0 \times 10^{8}$ cells $/ \mathrm{ml}$ of suspension. For subsequent measurements, we used a cell concentration in the middle of the range. Because the signal amplitude was relatively insensitive to cell concentration near its maximum value, small variations in the number of cells added did not influence the deformation data significantly. Thus, changes in DI reflected changes in the deformability of individual cells, rather than variations in the number of cells in the sample, such as might be present in clinical samples. A survey of 70 control subjects showed that although there was a $10 \%$ variation in the initial slope, the plateau of the deformation curve $\left(D I_{\max }\right)$ obtained under these optimum conditions varied no more than $3 \%$ from subject to subject. Repeated measurements on the same individuals were identical, showing that these differences reflected individual variations. Similarly, measurements with various animal cells of various sizes (mean cell volume $=45-100 \mu \mathrm{m}^{3}$ ) showed little influence of cell volume on the optimum cell concentration.

Once the optimum values of dextran and erythrocyte 


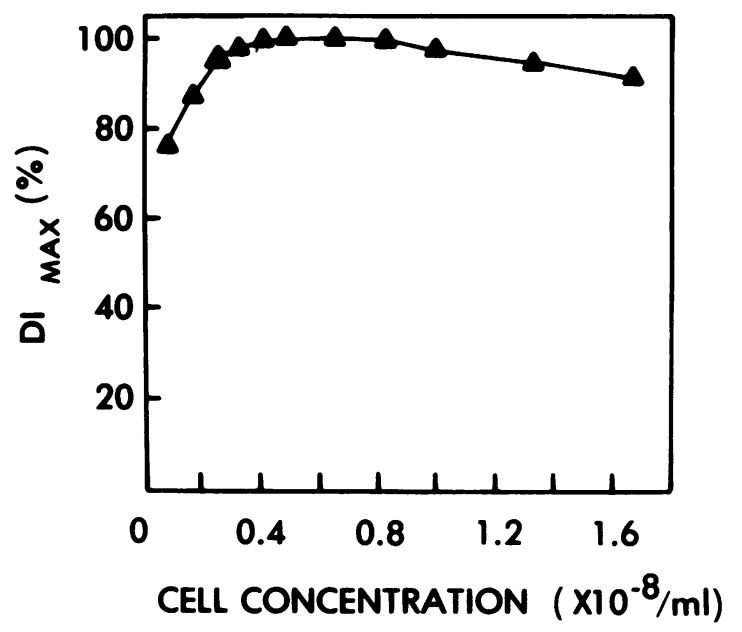

FIGURE 6 Maximum DI for normal cells as a function of cell concentration. Dextran concentration here and in all subsequent figures was $25 \mathrm{~g} / 100 \mathrm{ml}$ PBS.

concentrations were found, we studied the effect of medium osmolality, which in turn influences cell water content and internal viscosity, as well as cell geometry. Fig. 7A summarizes the behavior of normal cells in the range of 75-450 mosmol $/ \mathrm{kg}$. In the hypertonic media, maximum deformation, as measured by the plateau of the DI curve, was less than at $290 \mathrm{mosmol} / \mathrm{kg}$. This change was consistent with the increase in MCHC of the hypertonically dehydrated cells. The deformability remained at a relatively constant maximum value between 290 and $250 \mathrm{mosmol} / \mathrm{kg}$, and then progressively decreased as the cells became hypotonically swollen, to a minimum value at $\sim 125 \mathrm{mosmol} / \mathrm{kg}$. Below 125 mosmol $/ \mathrm{kg}$, the DI plateau increased once more, presumably because of leakage of intracellular ions and water into the low tonicity medium. At osmolalities between 175 and $125 \mathrm{mosmol} / \mathrm{kg}$, where osmotic lysis normally occurs, the shape of the deformation curve was altered (Fig. 7B). At very low shear stress, the value of DI rose normally but then it leveled off abruptly at the plateau, without the gradual asymptotic approach seen at higher osmolalities. However, on the second upward phase of the deformation vs. osmolality curve, below $125 \mathrm{mosmol} / \mathrm{kg}$, where substantial loss of intracellular volume had occurred, the deformation curves once again showed a gradual approach to the plateau values. Thus, it appears that the abrupt change in slope for normal cells in 175-125 mosmol/ $/ \mathrm{kg}$ medium signaled the approach to critical hemolytic volume.

Deformation of modified normal cells. Cells treated with increasing concentrations of LPC deformed almost normally in isotonic medium, except for cells exposed to high prelytic concentrations of LPC. However, in hypotonic medium, cell deformability was abnormally reduced, with increasing effect as the LPC concentration was increased (Fig. 8). Except for very
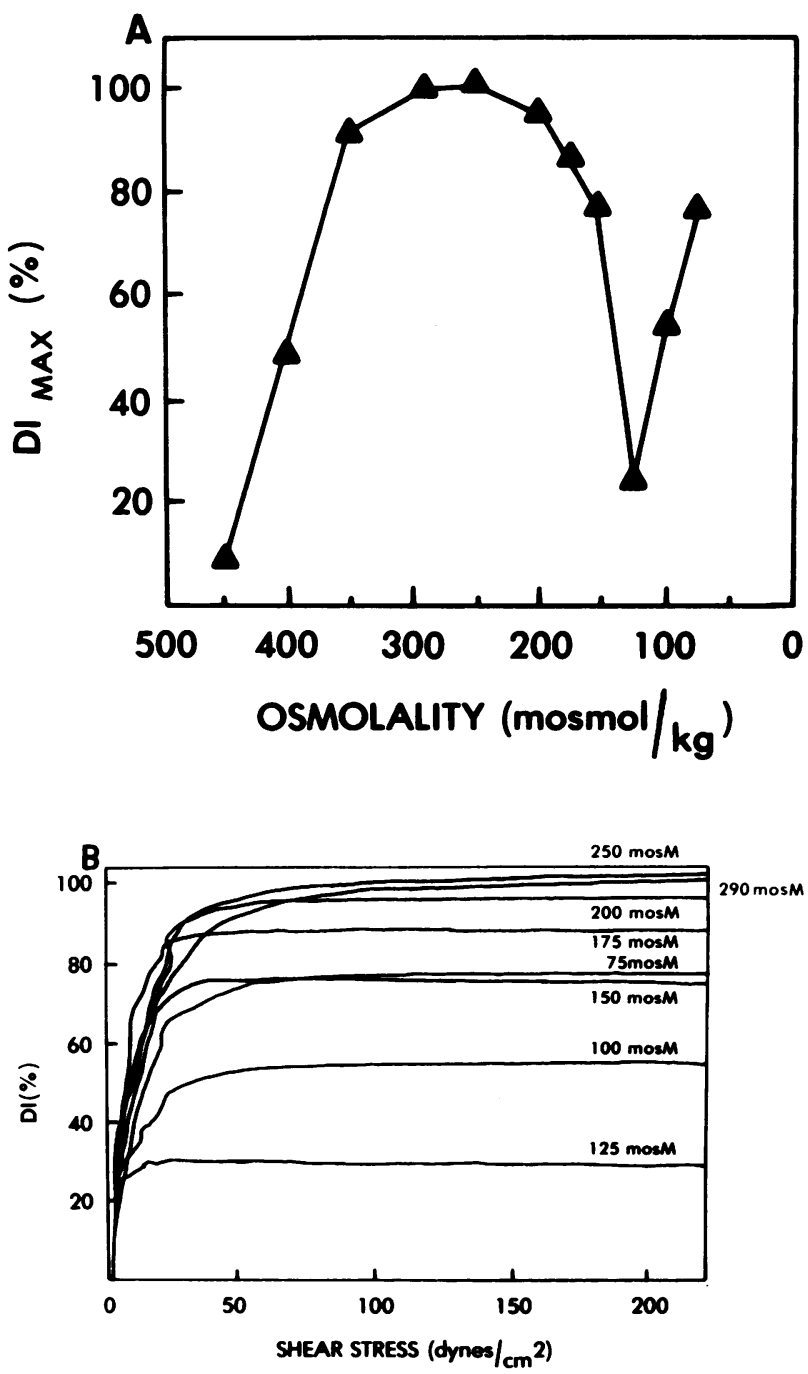

Figure 7 A. Maximum DI for normal cells as a function of suspending medium osmolality. B. DI vs. shear stress at various osmotic strengths. Note the flattening of the curves of osmolalities between 175 and $125 \mathrm{mosmol} / \mathrm{kg}$.

slight reductions in the initial slope of the DI vs. shear stress curve at high LPC concentrations, the shape of the curves in isotonic medium was similar to that for normal cells; the major change was a decrease in maximum deformation in hypotonic medium. Abrupt slope changes, as seen for normal cells between 175 and $125 \mathrm{mosmol} / \mathrm{kg}$, were found at higher osmotic strengths for LPC-treated cells, further supporting the association of this curve shape with the onset of osmotic cell lysis. A progressive increase in osmotic fragility with increasing concentrations of LPC showed that reduced surface area was the primary alteration in LPC-treated cells. Moreover, density gradient centrifugation confirmed the absence of measurable changes in cell water content. 


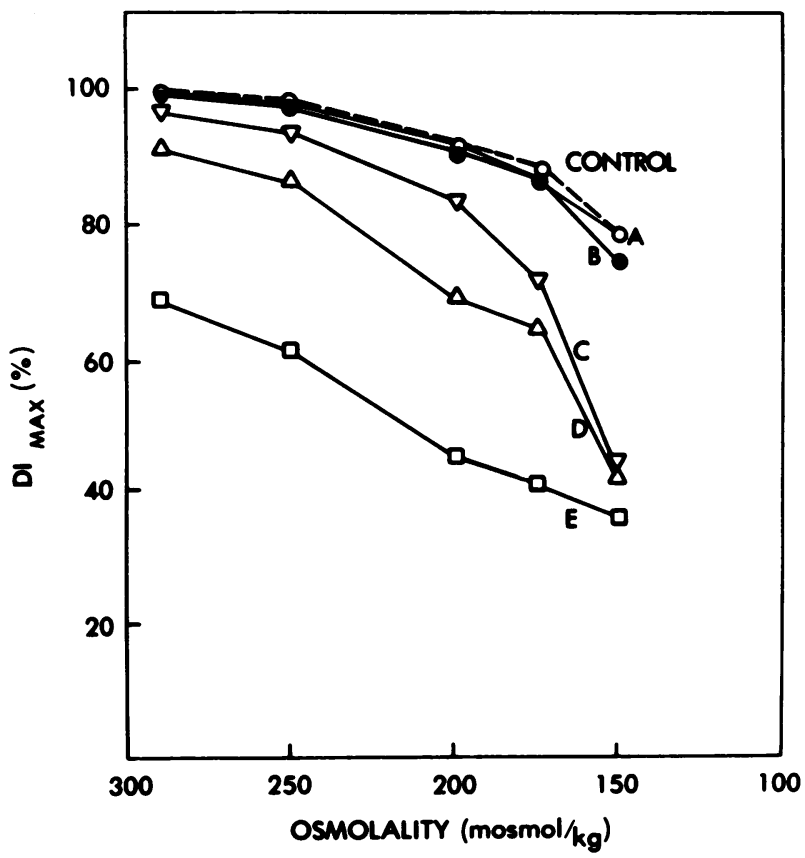

FIGURE 8 Maximum DI vs. osmolality for LPC-treated cells. Note premature decrease of deformability, especially at lower osmolality and higher LPC concentration. A, O, 1.0 $\mu \mathrm{mol} \mathrm{LPC} / \mathrm{ml}$ cells; B, O, $1.5 \mu \mathrm{mol} \mathrm{LPC} / \mathrm{ml}$ cells; C, $\nabla, 2.0$ $\mu \mathrm{mol} / \mathrm{ml}$ cells; $\mathrm{D}, \Delta, 2.5 \mu \mathrm{mol} / \mathrm{ml}$ cells; $\mathrm{E}, \square, 5.0 \mu \mathrm{mol} / \mathrm{ml}$ cells.

In contrast to LPC-treated cells, cells dehydrated with nystatin showed marked reductions in isotonic deformability. A graph of maximum DI vs. osmolality from 300 to $125 \mathrm{mosmol} / \mathrm{kg}$ showed the same upward trend as the corresponding curve for normal cells in the osmolality range from 450 to $290 \mathrm{mosmol} / \mathrm{kg}$ (Fig. 9). This behavior is consistent with a major influence of increased MCHC as a result of water loss and an attendant profound increase in internal viscosity. This suggested loss in cell water was confirmed by appropriate decreases in osmotic fragility and corresponding increases in MCHC and cell density as measured on Stractan gradients. Unlike the membrane-deficient LPC-treated cells, the dehydrated cells regained all the deformability of normal cells when they were subsequently suspended in hypotonic solutions. The osmotic strength at which normal deformability was restored correlated closely with the isotonic MCHC. In fact, the MCHC at the osmotic strength that restored normal deformability was always close to the normal MCHC of $35 \mathrm{~g} / \mathrm{dl}$. The slope of the DI vs. shear stress curve exhibited a more gradual approach to the DI plateau for samples in which the MCHC was still substantially elevated ( $>40 \mathrm{~g} / \mathrm{dl}$ ) under the conditions of deformability measurement. However, when the maximum DI was restored to the normal level in hypotonic medium, the initial portion of the DI curve was also restored to its normal shape. Moreover, cells treated with nystatin in isotonic medium, to maintain normal MCHC and water content, showed deformability behavior identical to that of normal untreated cells. Thus, nystatin itself had no effect on the cell deformability. Cell morphology was normal except for a slight flattening of severely dehydrated cells consistent with their reduced water content.

Cells heated to $47-48^{\circ} \mathrm{C}$, previously shown to have an increase in membrane shear modulus (20), exhibited minimal change in osmotic fragility and only modest reductions in the maximum deformation with increased duration of heating. This small decrease in deformation at maximum shear stress appeared to result from a very slight dehydration effect that occurred during heating. Density gradient centrifugation showed a slight increase in cell density that was too small to cause a measurable shift in osmotic fragility. The most notable feature of the DI vs. shear stress curves for the heated cells was a reduction in the initial slope, producing a more gradual approach to the maximum DI (Fig. 10). This effect of reduced deformation at low shear stress increased progressively as the heating period was prolonged. Furthermore, in contrast to the similar slope change noted in severely dehydrated cells, the abnormal slope for heated cells could not be returned to normal by reducing the suspending medium osmolality. In fact, reduction of medium osmolality brought the DI plateau for cells that were heated for 2-15 min almost into coincidence, with virtually

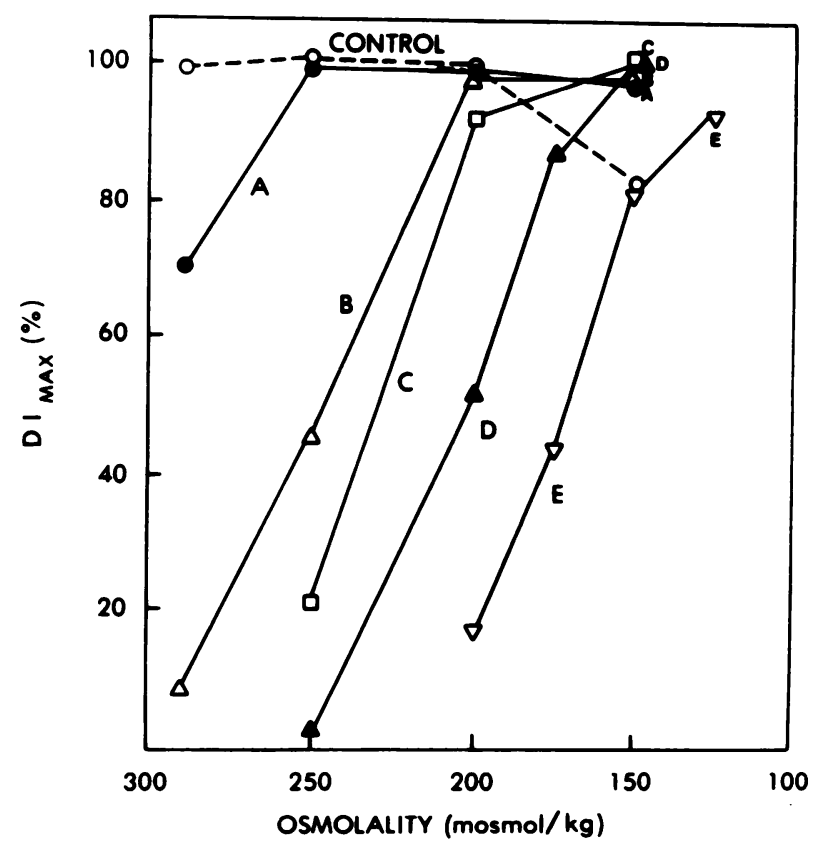

FIGURE 9 Maximum DI vs. osmolality for dehydrated cells. Samples A through $E$ were increasingly dehydrated. Note marked upward slope in contrast to that in Fig. 8. 


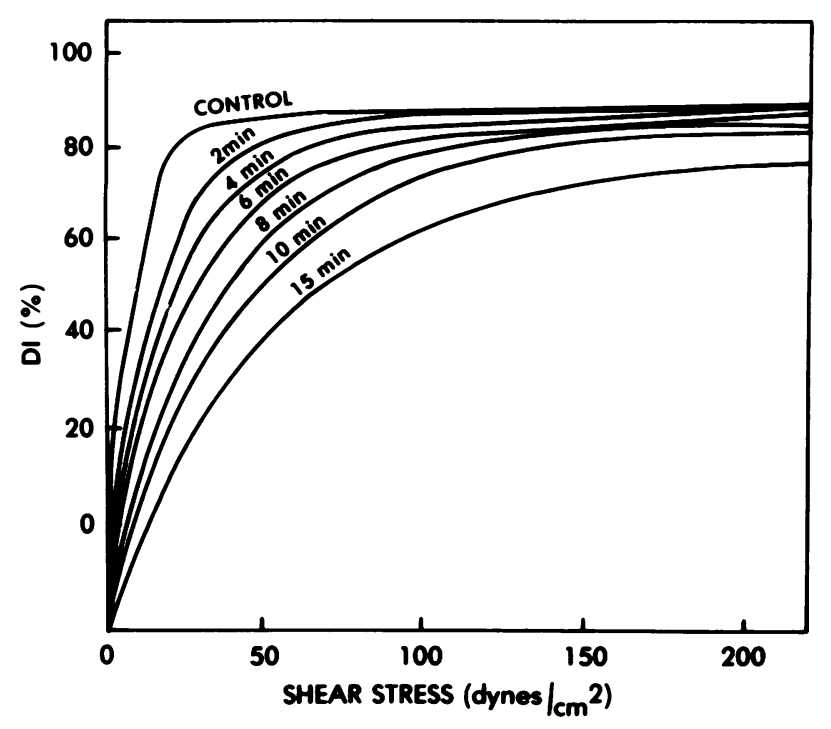

Figure 10 DI vs. shear stress for cells heated to $47^{\circ} \mathrm{C}$ for the indicated periods. Note characteristic reduction in deformation at low and moderate shear stress.

no change in initial slope. Cell morphology was identical to that of unheated normal cells, confirming the absence of fragmentation.

The apparent effect of increased membrane shear modulus on the DI vs. shear stress curve shown by heated cells was further studied using diamide-treated cells. Others have shown that treatment with low concentrations of diamide induced cross-linking of the membrane-associated protein spectrin and an increase in membrane shear modulus (21). The DI vs. shear stress curves for cells incubated in $0.10-0.50 \mathrm{mM}$ diamide for $60 \mathrm{~min}$ were closely similar to the curves for cells heated to $47.5^{\circ} \mathrm{C}$ for periods ranging up to 15 min. As in heated cells, a slight degree of dehydration appeared to occur at the higher diamide concentrations. Also, reduction of the suspending medium osmolality resulted in normalization of the maximum DI without alteration of the reduced initial slopes.

Application to hemolytic disorders. Blood from patients with hereditary spherocytosis was separated into subpopulations of increasing cell density. Whole blood and the cell subpopulations were then suspended in dextran solutions at various osmotic strengths for ektacytometric measurement. A graph of maximum deformation as a function of osmotic strength is shown for the various subpopulations from one experiment in Fig. 11. The low density subpopulations from the upper portions of the gradient showed behavior similar to that of normal control cells, but with moderately reduced deformation as the osmolality was decreased in a manner similar to LPC-treated cells. On the other hand, the dense subpopulations behaved like the artificially dehydrated cells. That is, they deformed poorly in

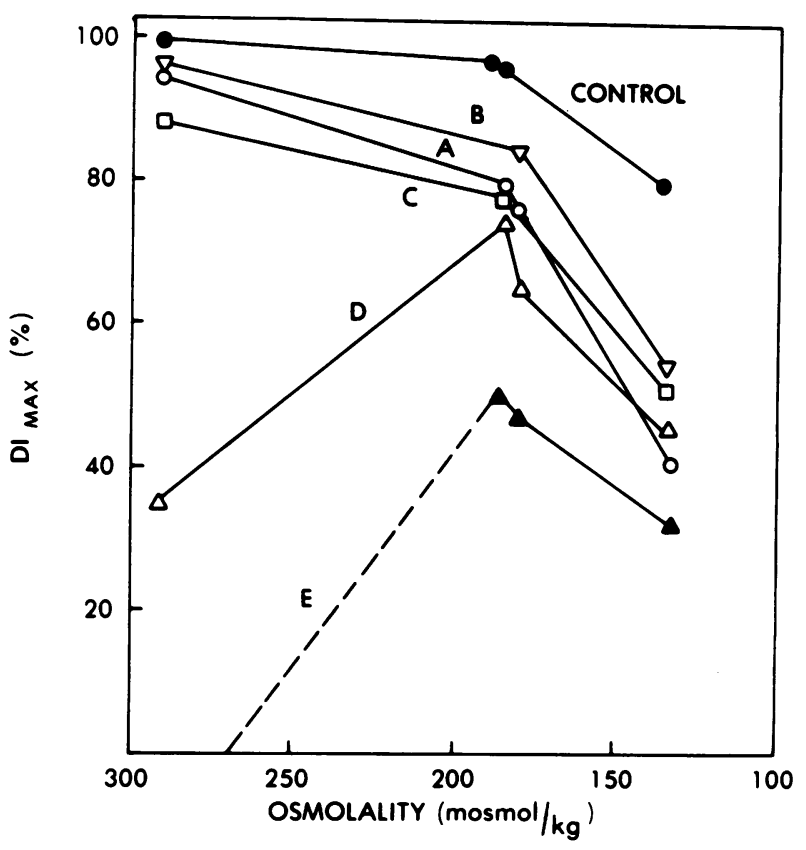

FIGURE 11 Maximum DI vs. osmolality for hereditary spherocytes separated on Stractan density gradients. Samples $A-E$ are designated in order of increasing density. Note change in slope of the initial part of the curve from downward to upward in the two most dense cell populations. Also note failure of all cells to attain normal DI maximum at any osmolality.

isotonic medium but became more deformable as the osmolality was decreased. These cells reached a maximum deformability that was less than that of all the other subpopulations and control cells. As the osmolality was further reduced, the deformability of these high MCHC spherocytes decreased, unlike the behavior of the dehydrated normal cells. All subpopulations of hereditary spherocytes, even those with high MCHC, showed considerable increases in osmotic fragility. All changes in the slope of the DI curve could be corrected by reduction of the medium osmolality. This suggested the absence of a substantial increase in the membrane shear modulus.

Cells from a patient with hemoglobin CC disease were studied as well. In contrast to the hereditary spherocytes, all gradient subpopulations of hemoglobin CC cells showed decreased deformation in isotonic medium and increasing deformation with decreasing osmolality (Fig. 12). Every subpopulation of cells deformed abnormally in isotonic medium. However, when the osmolality was reduced to $100 \mathrm{mosmol} / \mathrm{kg}$, the deformability was restored to a virtually normal maximum value in all subpopulations. This evidence for cellular dehydration, which is well known to be characteristic of hemoglobin CC cells $(25,26)$, was confirmed by a marked reduction in osmotic fragility for all the hemoglobin CC cells. Again, the initial slope 


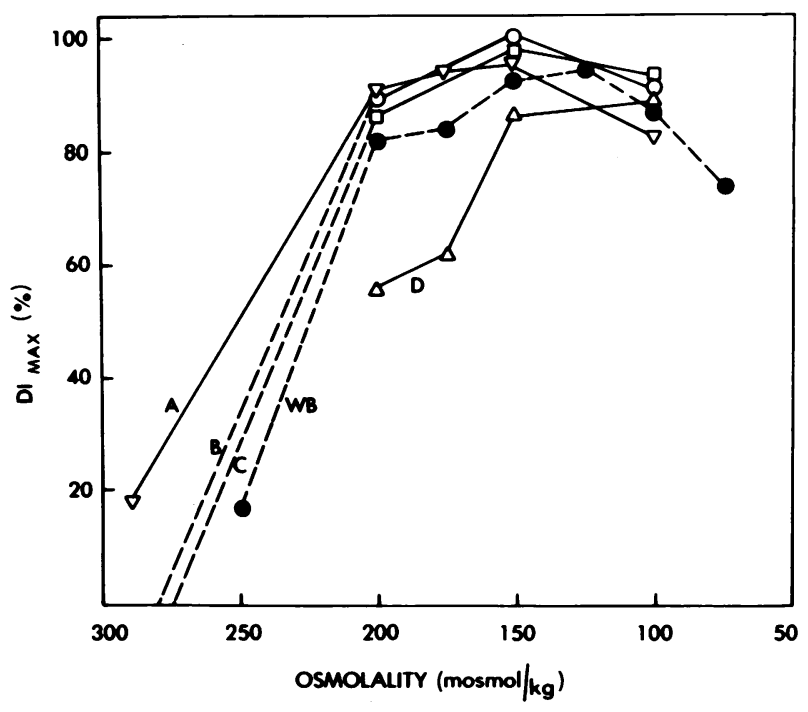

FIGURE 12 Maximum DI vs. osmolality for hemoglobin CC cells separated on Stractan gradients. Note marked increase in deformability with decreasing osmolality for all cell subpopulations. A-D are in order of increasing density; WB is whole blood. Also note, in contrast to hereditary spherocyte cells, DI maximum approaches $100 \%$. A, $\nabla$; B, O; C, $\square$; D, $\Delta$; whole blood, $\bigcirc$.

of the DI curves was normal when normal deformability was restored by suspending the cells in hypotonic medium, suggesting normal membrane deformability.

Blood from a patient with hereditary pyropoikilocytosis, a hemolytic anemia characterized by increased thermal instability of the erythrocyte membranes (27), was also studied. The whole blood showed a marked reduction in deformability in isotonic medium. The most deformable, least dense gradient subpopulations had a maximum DI of $64 \%$ (normal $=100 \%$ ), and succeeding populations had $\mathrm{DI}_{\max }$ values of 62,47 , and $1 \%$. Reduction of the medium osmolality to $225 \mathrm{mosmol} / \mathrm{kg}$ made all the cells less deformable except the bottom gradient subpopulations. With further reduction of osmolality to $175 \mathrm{mosmol} / \mathrm{kg}$, the deformability of the bottom subpopulations also decreased. These observations, supported by an increase in osmotic fragility for these cells, were consistent with the demonstrated membrane loss by these unstable cells. The slopes of the DI curves all showed a gradual approach to $\mathrm{DI}_{\max }$ that was not improved by reduction of the suspending medium osmolality. However, neither was there restoration of a normal $\mathrm{DI}_{\max }$. Thus, it was not possible to determine whether abnormal membrane elasticity was also present.

\section{DISCUSSION}

These studies represent a new approach to the analysis of erythrocyte deformability that combines four useful features. First, changes in osmotic strength permitted the identification of increased internal viscosity and reduced $S / V$ as causes of reduced deformability. Second, individual alteration of the internal viscosity, $\mathrm{S} / \mathrm{V}$, and membrane shear modulus of normal cells permitted characterization of the influence of each alteration upon ektacytometric measurements. Third, separation of patient blood samples on density gradients provided homogeneous samples for the ektacytometer and permitted the assessment of heterogeneity between subpopulations. Finally, use of the ektacytometer with its small sample requirement and continuous regulation of shear stress permitted measurements over a range of osmotic and shear stress values for the small gradient subpopulations.

The use of the new photometric analysis system now makes feasible the analysis of numerous blood samples with abnormal deformability. These studies have demonstrated that the DI function obtained so conveniently with this modification does in fact provide an accurate measurement of cell deformability. Variation in the value of the $\mathrm{DI}_{\max }$ plateau with osmolality provides a simple test for the identification of decreased S/V or increased intracellular viscosity. For cells with a decreased $\mathrm{S} / \mathrm{V}$, the curve of $\mathrm{DI}_{\max }$ vs. osmolality had an exaggerated downward slope, reflecting the limitation of deformability as the cells prematurely approached spherical geometry in hypotonic medium. For cells with increased internal viscosity, this curve had a contrasting and characteristic upward slope, reflecting the increase of intracellular fluidity by hypotonic dilution of the hemoglobin. The effects of increased membrane shear modulus were revealed in the curve showing DI (not $\mathrm{DI}_{\max }$ ) as a function of shear stress. For cells with increased membrane shear modulus this curve had a reduced initial slope, reflecting membrane resistance to cell deformation at low shear stress. With a flow channel device, Rakow and Hochmuth (20) have previously shown that increased membrane shear modulus reduces the extent of cell elongation only at low shear stress. This direct observation supports our interpretation that a reduction in the initial slope of a DI curve with a normal $\mathrm{DI}_{\max }$ plateau indicates increased membrane shear modulus. However, this criterion can be applied only in the presence of a near-normal $\mathrm{DI}_{\max }$ value. Thus, it cannot be used for cells with severe membrane deficiency.

Our studies of artificially modified normal cells have provided a basis for the interpretation of the deformability curves obtained for cells from patients with three different hemolytic disorders. In hereditary spherocytosis, all subpopulations of cells exhibited the absolute limitation in maximum deformation characteristic of cells deficient in membrane surface area. In addition, a varying proportion of cells also showed the characteristic increase in deformability with de- 
creasing osmolality expected for high MCHC cells. The minimal changes in the initial slope of the DI curves were no greater than those seen for LPC-treated model cells. From this we conclude that there were no appreciable changes in membrane shear modulus in the hereditary spherocytes. This is of particular interest because an increase in the membrane shear modulus has previously been proposed as a substantial source of reduced deformability in hereditary spherocytes (28), a suggestion that has been popularly accepted. However, Nakashima and Beutler (29) have recently reported normal membrane deformability in hereditary spherocytes. Furthermore, a dominant effect of reduced surface area on cell survival was indicated in studies by Cooper and Jandl (30). Additional effects of high MCHC have also been suggested (31).

In contrast to hereditary spherocytes, all cell subpopulations from a patient with hemoglobin CC disease showed a defect in deformability that was dominated by decreased water content and consequent increased internal viscosity. This observation is supportive of earlier studies by Charache et al. (25) and by Murphy (26), showing cellular dehydration in this disorder. We found no evidence for cells with a reduced S/V.

All subpopulations of hereditary pyropoikilocytes were markedly undeformable in isotonic medium. A further reduction of deformability in slightly hypotonic medium characterized most of the cells as being severely membrane deficient. Because none of the cell populations could be restored to normal deformability, it was not possible to determine whether decreased membrane elasticity also contributed to the deformability defect.

These studies provide a first approach to the systematic identification of specific defects underlying abnormal deformability in pathologic erythrocytes. Extension of this approach should help define the physiologic importance of the three major factors that influence whole cell deformability. It may also identify the primary causes of shortened erythrocyte survival in hemolytic disorders.

\section{ACKNOWLEDGMENTS}

This work was supported in part by U. S. Public Health Service grants AM 16095, AM 26263, and HL-20985, and by a grant from Institut National de la Sante et de la Recherche Medicale (U048), France. This is publication 17 from the MacMillanCargill Hematology Research Laboratory, University of California, San Francisco, Calif.

\section{REFERENCES}

1. Weed, R. I. 1970. The importance of erythrocyte deformability. Am. J. Med. 49: 147-150.

2. LaCelle, P. L. 1970. Alteration of membrane deformability in hemolytic anemias. Semin. Hematol. 7: 355-371.

3. Jacob, H. S. 1974. Pathologic states of the erythrocyte membrane. Hosp. Pract. 9(12): 47-53.
4. Mohandas, N., W. M. Phillips, and M: Bessis. 1979. Red blood cell deformability and hemolytic anemias. Semin. Hematol. 16: 95-114.

5. Teitel, P. 1977. Basic principles of the "Filterability Test" (FT) and analysis of erythrocyte flow behaviour. Blood Cells. 3: 55-70.

6. Usami, S., S. Chien, and J. F. Bertles. 1975. Deformability of sickle cells as studied by microsieving. J. Lab. Clin. Med. 86: 274-279.

7. Chien, S., S. Usami, and J. F. Bertles. 1970. Abnormal rheology of oxygenated blood in sickle cell anemia.J. Clin. Invest. 49: 623-634.

8. Weed, R. I., P. L. LaCelle, and E. W. Merrill. 1969. Metabolic dependence of erythrocyte deformability. J. Clin. Invest. 48: 796-809.

9. Evans, E. A., and P. L. LaCelle. 1975. Intrinsic material properties of the erythrocyte membrane indicated by mechanical analysis of deformation. Blood. 45: 29-43.

10. Hochmuth, R. M., N. Mohandas, and P. L. Blackshear, Jr. 1973. Measurement of the elastic modulus for red cell membrane using a fluid mechanical technique. Biophys. J. 13: 747-762.

11. Bull, B. S., and J. D. Brailsford. 1975. A new method of measuring the deformation of the red cell membrane. Blood. 45: 581-586.

12. Leblond, P. F., and L. Coulombe. 1979. The measurement of erythrocyte deformability using millipore membranes. A sensitive technique with clinical application. J. Lab. Clin. Med. 94: 133-143.

13. Chien, S. 1977. Principles and techniques for assessing erythrocyte deformability. Blood Cells. 3: 71-99.

14. Bessis, M., and N. Mohandas. 1975. A diffractometric method for the measurement of cellular deformability. Blood Cells. 1: 307-313.

15. Allard, C., N. Mohandas, and M. Bessis. 1977. Red cell deformability changes in hemolytic anemias estimated by diffractometric methods (ektacytometry). Blood Cells. 3: 209-221.

16. Smith, J. E., N. Mohandas, and S. B. Shohet. 1979. Variability in erythrocyte deformability among various mammals. Am. J. Physiol. 236: H725-H730.

17. Bessis, M., and N. Mohandas. 1975. Deformability of normal, shape-altered and pathological red cells. Blood Cells. 1: 315-321.

18. Bessis, M. 1973. Red cell shapes. An illustrated classification and its rationale. In Red Cell Shape. M. Bessis, R. I. Weed, and P. F. Leblond, editors. Springer-Verlag New York, Inc., New York. 1-25.

19. Clark, M. R., N. Mohandas, V. Caggiano, and S. B. Shohet. 1978. Effect of abnormal cation transport on deformability of desiccytes. J. Supramol. Struct. 8: 521-532.

20. Rakow, A. L., and R. M. Hochmuth. 1975. Effect of heat treatment on the elasticity of human erythrocyte membrane. Biophys. J. 15: 1095-1100.

21. Fischer, T. M., C. M. W. Haest, M. Stohr, D. Kamp, and B. Deuticke. 1978. Selective alteration of erythrocyte deformability by SH-reagents. Evidence for an involvement of spectrin in membrane shear elasticity. Biochim. Biophys. Acta. 510: 270-282.

22. Clark, M. R., R. C. Unger, and S. B. Shohet. 1978. Monovalent cation composition and ATP and lipid content of irreversibly sickled cells. Blood. 51: 1169-1178.

23. Coulombel, L., G. Tchernia, and N. Mohandas. 1979. Human reticulocyte maturation and its relevance to erythropoietic stress. J. Lab. Clin. Med. 94: 467-474.

24. Fischer, T. M., M. Stohr, and H. Schmid-Schonbein. 1978. Red blood cell microrheology: comparison of the behavior of single RBC and liquid droplets in shear flow. 
American Institute of Chemical Engineers Symposium Series. 74: 38-45.

25. Charache, S., C. L. Conley, D. F. Waugh, R. J. Ugoretz, and J. R. Spurrell. 1967. Pathogenesis of hemolytic anemia in homozygous hemoglobin C disease. J. Clin. Invest. 46: 1795-1811.

26. Murphy, J. R. 1968. Hemoglobin CC disease: rheological properties of erythrocytes and abnormalities in cell water. J. Clin. Invest. 47: 1483-1495.

27. Zarkowsky, H. S., N. Mohandas, C. B. Speaker, and S. B. Shohet. 1975. A congenital haemolytic anaemia with thermal sensitivity of the erythrocyte membrane. Br. J. Haematol. 29: 537-543.
28. LaCelle, P. L., and R. I. Weed. 1969. Reversibility of abnormal deformability and permeability of the hereditary spherocyte. Blood. 34: 858a. (Abstr.)

29. Nakashima, K., and E. Beutler. 1979. Erythrocyte cellular and membrane deformability in hereditary spherocytes. Blood. 53: 481-485.

30. Cooper, R. A., and J. H. Jandl. 1969. The role of membrane lipids in the survival of red cells in hereditary spherocytosis. J. Clin. Invest. 48: 736-744.

31. Erslev, A. J., and J. Atwater. 1963. Effect of mean corpuscular hemoglobin concentration on viscosity. J. Lab. Clin. Med. 62: 401-406. 